by wearing translucent goggles. so that one eye saw only a field of horizontal stripes and the other only a field of vertical stripes. In the electrophysiological experiments the orientationsensitive neurones that were found responded to the orientation of rearing for the eye being tested and these cells could typically not be affected by stimuli shown to the other eye. Nevertheless, behaviourally Hirsch found that in these animals discrimination of shapes and orientations trained entirely with one eye could be performed (though somewhat less well) when tested on the other.

The findings of Hirsch with discrimination of orientations around vertical and around horizontal are perhaps the clearest. Here cats tested with the eye reared on vertical stripes performed better than with the eye reared with horizontal stripes in the discrimination of orientations around vertical. Similarly when using the eyes reared with horizontal stripes and tested around horizontal they were better than when using those reared with vertical. And cats reared normally did a little better still.

The important finding, however, was that differences between the groups were rather small and cats did manage to perform discriminations between those orientations for which they evidently lacked cortical neurones. Further electrophysiological study of these same cats following the behavioural experiments confirmed that the deficit of neurones sensitive to orientations other than those with which they were raised still existed.

One might have supposed that orientation-sensitive neurones would at least be used in making discriminations between lines of different orientation, but perhaps even this must be rethought. One cannot, on the basis of these experiments, immediately dismiss the idea of neurones in the visual cortex as feature detectors, but once again the brain seems to be more subtle and versatile than one would have given it credit for.

\section{POPULATION ECOLOGY \\ Fighting a Forest Pest}

from our Soviet Correspondent

A NEW mathematical theory of insect population dynamics may facilitate the preservation of forests from xylophagic insects without the excessive use of insecticides, which may themselves be harmful to standing timber. This approach, developed by A. S. Isaev, of the Sukachev Forestry Institute, and R. G. Khlebopros, of the Siberian Branch of the Soviet Academy of Sciences (Dokl. Akad. Nauk SSSR, 208, $225 ; 1973)$, is based on the concept of a "critical" population density at which ecological balance breaks down and the establishment of the "multiplication factor"-the ratio of insect density in the $(n+1)$ th generation to that in the $n$ th.

Isaev and Khlebopros estimated the multiplication factor as a function of population density itself, the quantity and type of food, various external factors independent of the population density and the lag in the natural regulatory processes of forest ecology. They found that, for a constant type of food, a stability level should be established, around which population density should fluctuate in a manner dependent on the availability of that food. But as certain types of insect, notably the horned beetle Monochamus urussovi, increase in population density, regulation of the population by overconsumption of their food does not occur; above a certain "critical" density, the feeding habits of such insects become modified to include a great range of plants. When this "critical" density is exceeded, the possibility of a second stability level of population in equilibrium with food supplies does, of course, exist ; in practice, however, this will not occur until there has been a massive disruption of the ecology and widespread devastation of the forest, such as Monochamus urussovi has produced in recent years in the fir forests of Central Siberia.

To remedy this situation, in localities where the local population density of insects has increased above the critical level, Isaev and Khlebopros suggest the use not of insecticides but of insect repellents which, without harming the trees, would disperse the insects into surrounding areas and thus reduce the population density below the critical level. This would create a situation in which the natural regulating factors of forest ecology would gradually reduce the density of the population.

\section{OCEANOGRAPHY}

\section{Polygons and Waves}

from a Correspondent

LONGUET-HigGins, of the University of Cambridge, has discovered a new and remarkably simple approximation for the profile of the steepest possible gravity waves which can occur in deep water (Proc. Roy. Soc. Lond., A331, $445 ; 1973)$. The usual conditions for a gravity wave are that the fluid be inviscid, incompressible and irrotational, that surface particles move with the surface, and that the pressure be constant over the surface. Expressed mathematically, these conditions give rise to non-linear equations which, strictly speaking, have never been completely solved.

The simplest approximation is to assume that the slope of the surface is everywhere very small (say less than 1 in 10). One may then ignore the nonlinearities, and obtain the well-known

\title{
More about Aerodynamics of Buildings
}

ONE of the more important things that have to be taken into account when designing a building is how the structure will behave in a wind. To this end many people have in the past examined what happens when cylinders of square cross-section, typifying many buildings or their component parts, are exposed to rapid air flow in a wind tunnel.

In Nature Physical Science next Monday (February 26) Mulhearn describes such an experiment which he carried out in the 4 foot by 5.5 foot closed-circuit wind tunnel in the Engineering Laboratories at the University of Cambridge. $\mathrm{He}$ used a cylinder with 6-inch sides which was exposed to a wind of velocity 70 foot $^{-1}$ (about 50 mile h $^{-1}$ ).

Earlier investigators, such as Vickery, established that the resistance of a square cylinder to wind decreases when the flow becomes turbulent. Vickery contended that turbulence promotes reattachment of the flow, which originally separated from the leading face of the cylinder (see diagram), at a position closer to the leading edge than is the case for non-turbulent flow. Bearman and Kavur, however, found that no reattachment occurred in cases where the angle of incidence $\alpha$ was small.

Mulhearn's observations neatly suggest a compromise, in that he finds no reattachment at small angles of incidence but that the phenomenon does occur at larger values. $\mathrm{He}$ has confirmed the earlier finding that reattachment occurs sooner when the flow is turbulent, and he says that this implies that, even when reattachment does not occur, the shear layers emanating from the leading corner are closer to face A.

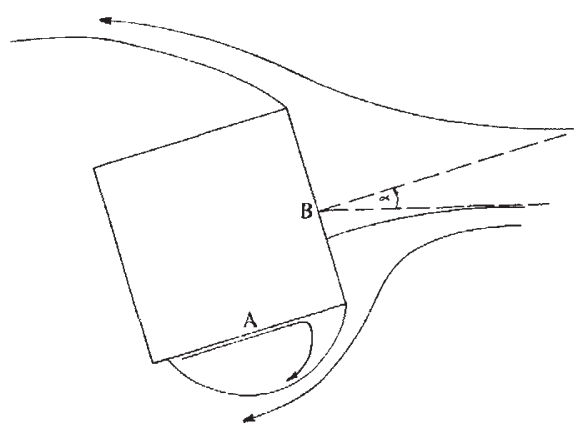

What is still not clear, however, is why resistance should be decreased as the separated shear layer comes nearer to the surface. 